\title{
Liposarcoma of the spermatic cord: a report of two cases
}

\section{CJ Tan, BVM Dasari, J Smyth, RJ Brown}

\section{Daisy Hill Hospital, Newry, UK}

\section{ABSTRACT}

Liposarcomas of the spermatic cord are unusual and rarely reported in the literature. These tumours can sometimes be mistaken for the common scrotal swellings such as hydrocoeles and hernias. Careful clinical and radiological examination will help in appropriate preoperative planning and surgery by an experienced surgical team. We report our experience of two cases of such scrotal swellings.

\section{KEYWORDS}

Liposarcoma - Spermatic cord - Neoplasms

Accepted 29 March 2011; published online 20 December 2011

\section{CORRESPONDENCE TO}

Chuan Tan, Surgical Assessment Unit, Daisy Hill Hospital, 5 Hospital Road, Newry BT35 8DR, UK

E: cjtan99@hotmail.com

Paratesticular neoplasms account for only $2 \%$ of scrotal neoplasms and most are benign. Malignant paratesticular tumours are commonly sarcomas and $3-7 \%$ of these are liposarcomas. ${ }^{1}$ Hence, liposarcomas of the spermatic cord are unusual and rarely reported in the literature. These tumours can sometimes be mistaken for the more common scrotal swellings such as hydrocoeles and hernias. Careful clinical and radiological examination will help in appropriate preoperative planning and surgery by an experienced surgical team. We report two cases of such scrotal swellings.

\section{Case History 1}

A 62-year-old man presented to a surgical clinic with a large scrotal swelling on the left side. Initial clinical impression was that of a large hydrocoele. However, ultrasonography was inconclusive of a hydrocoele. Computed tomography (CT) showed a $23 \mathrm{~cm} \times 18 \mathrm{~cm}$ scrotal mass containing multiloculated foci of fatty attenuation with soft tissue septa (Fig 1). There were multiple soft tissue components and a normal left testicle could not be identified. Tumour markers were normal.

The possible diagnosis of a testicular teratoma was made. A radical left orchidectomy was performed through the groin. A thick $4 \mathrm{~cm}$ spermatic cord was visualised and ligated. Histopathological studies revealed an encapsulated $225 \mathrm{~mm} \times 256 \mathrm{~mm} \times 110 \mathrm{~mm}$ fibro-fatty tumour encasing the testis. There was no evidence of primary testicular malignancy. This was a lipomatous tumour traversed by fibrous bands with a number of pleomorphic scattered lipoblasts. There was no necrosis, undifferentiated areas or vascular invasion. This was consistent with a well differentiated spermatic cord liposarcoma. There was no normal tissue beyond the tumour mass and therefore the surgical resection margin was positive.

Two-month postoperative follow-up CT showed abnormal soft tissue in the left inguinal region suggesting residual disease. The patient was referred to a specialist tertiary centre for wide local resection of his left inguinal canal where

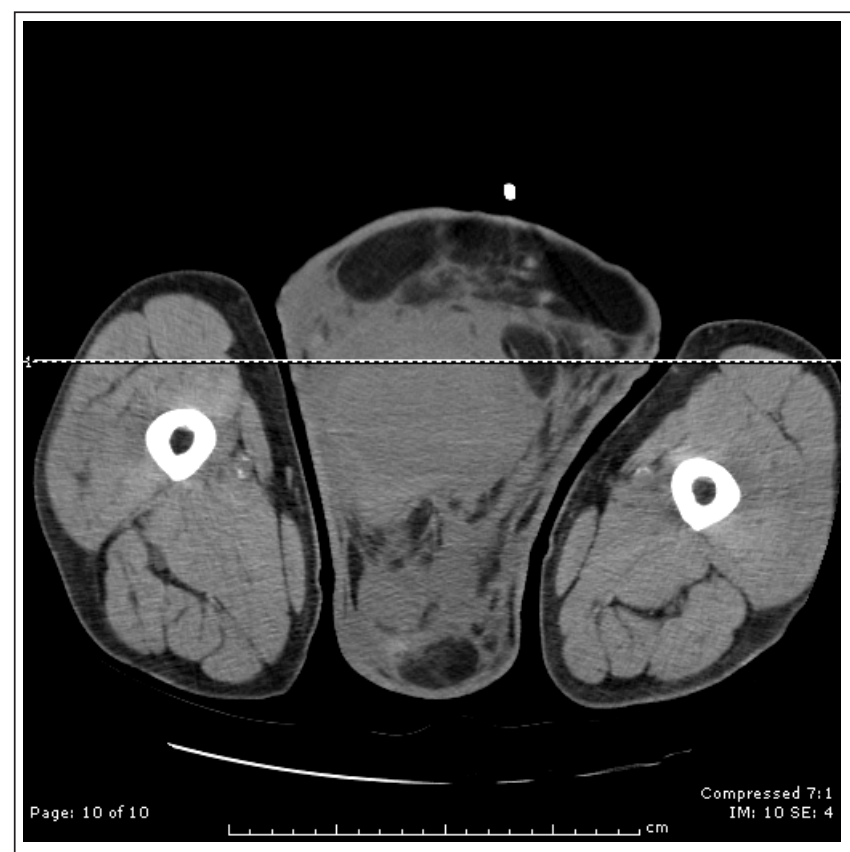

Figure 1 CT of the scrotum (axial view) demonstrating liposarcoma 
all the residual contents including the spermatic cord were excised. Retroperitoneal lymph node dissection was also performed. Histopathological study showed no residual disease. Incidentally, he was diagnosed to have a non-small cell adenocarcinoma in the left upper lobe of the lung and also underwent a left upper lobectomy for the lung lesion. The patient did not have any adjuvant radiotherapy or chemotherapy. He is currently disease free and follow-up CT at one year did not show any recurrence.

\section{Case History 2}

A 70-year-old man presented to the surgical outpatients department with a 1-year history of progressive, painless swelling in the left groin and scrotum (Fig 2). His past medical history included previous left inguinal hernia repair. Examination of the left scrotum revealed a large, firm, irregular swelling separate from the testis. The left testis felt normal.

Initial blood investigations including tumour markers were normal. Ultrasonography of the scrotum demonstrated inferior displacement of the left testicle by a hypervascular mass with a solid component. The right testis appeared normal. A radiological diagnosis of a suspicious paratesticular

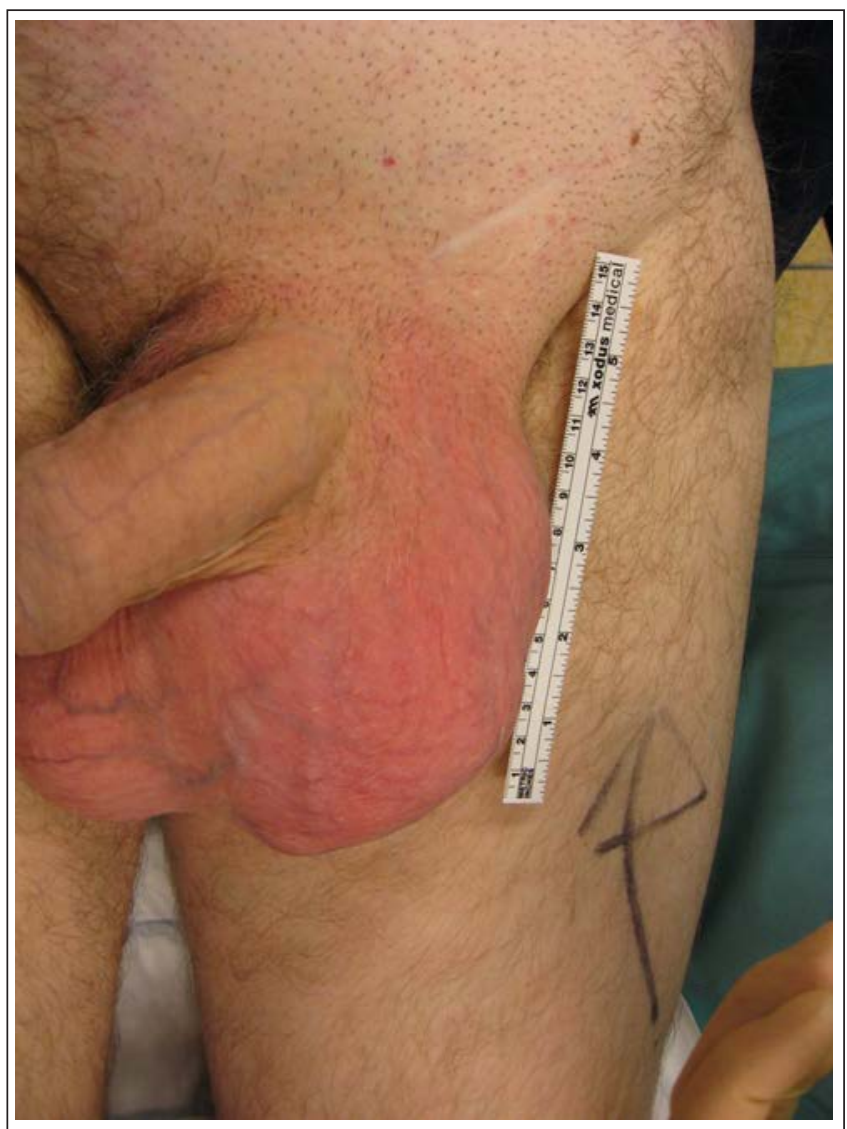

Figure 2 Large left inguinoscrotal swelling seen in patient

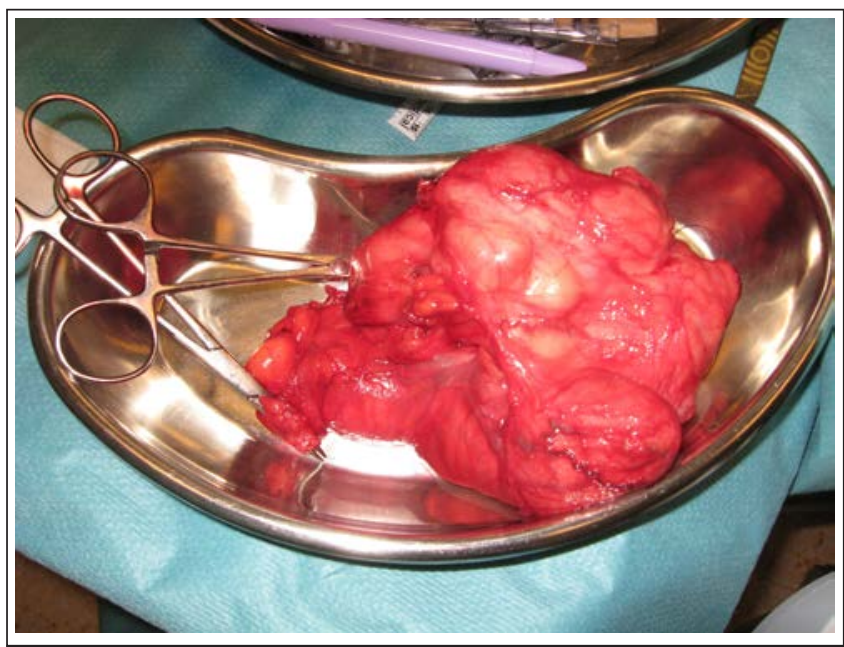

Figure 3 Complete resection specimen including left testicle

sarcoma was made. Staging CT of the chest, abdomen and pelvis revealed no lymphadenopathy or metastases.

The patient underwent a left radical orchidectomy and wide local excision that proved technically difficult due to previous inguinal hernia repair. Nevertheless, complete macroscopic excision was achieved (Fig 3). Histological examination of the specimen showed a low grade sclerosing liposarcoma with a $4 \mathrm{~cm}$ clear resection margin. The patient did not have any adjuvant radiotherapy or chemotherapy and is currently disease-free.

\section{Discussion}

Only $0.1 \%$ of liposarcomas present as inguinal hernias. They often manifest as a painless, slow growing mass in the inguinal region. It is difficult to differentiate between benign lipomas and liposarcomas. Features suggestive of malignancy include rapid growth, large size and symptomatic presentation. While common inguinoscrotal swellings (hernias and hydrocoeles) can be diagnosed on clinical examination, all the atypical swellings should be further investigated before surgical exploration.

Ultrasonography is the modality of choice to detect and evaluate these paratesticular tumours.,5 Ultrasonography typically reveals a solid, hyperechoic, heterogenous lesion separate from the testicle compared to benign lipomas, which are usually smaller and homogenous. CT usually shows a mass lesion of fat attenuation intermixed with nonlipomatous septa or soft tissue nodules. ${ }^{4}$ The advantage of radiological diagnosis cannot be overemphasised as demonstrated in the different outcomes of both our cases. A high radiological suspicion of liposarcoma directs the surgeon to perform a wide local excision, thus avoiding the need for further re-excisions and reducing the risk of disease recurrence.

Most spermatic cord liposarcomas are low grade, well differentiated tumours. They commonly spread via local invasion to adjacent structures. In high grade lesions, haema- 
togenous and lymphatic spread can occur. Surgical excision in the form of a radical orchidectomy with high ligation of the spermatic cord and wide local resection of tumour margins is mandatory and represents the mainstay of treatment options. ${ }^{2,3,5}$ It is important to differentiate spermatic cord liposarcomas from rhabdomyosarcomas as the latter carry an increased risk of lymphatic spread and retroperitoneal involvement, requiring retroperitoneal lymph node dissection. This has not been shown to be of benefit in management of cord liposarcomas. ${ }^{5}$

The risk of recurrence is high and in a 20-year case series of adult spermatic cord sarcomas there was a $55 \%$ recurrence rate reported. Commonly, these are local recurrences at the previous surgical site. Risk factors for recurrence include tumour grade, size, depth of invasion and, most importantly, surgical margin status. ${ }^{5}$ The use of adjuvant radiotherapy has been proposed to reduce local recurrence but no convincing large scale studies are avail- able. ${ }^{6}$ There is no evidence of any benefit of chemotherapy. Long-term follow up is essential due to the high recurrence rate.

\section{References}

1. Fitzgerald S, MacLennan GT. Paratesticular liposarcoma. J Urol 2009; 181: 331-332.

2. Cheng $\mathrm{YC}$, Chou $\mathrm{YH}$, Chiou HJ et al. Liposarcoma of the spermatic cord: a report of two cases and a review of the literature. J Med Ultrasound 2004; 12: 125-130.

3. Schwartz SL, Swierzewski SJ 3rd, Sondak VK, Grossman HB. Liposarcoma of the spermatic cord: report of 6 cases and review of the literature. J Urol 1995; 153: 154-157.

4. Bhosale PR, Patnana M, Viswanathan C, Szklaruk J. The inguinal canal: anatomy and imaging features of common and uncommon masses. Radiographics 2008; 28: 819-835.

5. Coleman J, Brennan MF, Alektiar K, Russo P. Adult spermatic cord sarcomas: management and results. Ann Surg Oncol 2003; 10: 669-675.

6. Ballo MT, Zagars GK, Pisters PW et al. Spermatic cord sarcoma: outcome, patterns of failure and management. J Urol 2001; 166: 1,306-1,310. 